\title{
Progranulin
}

\section{Functions and neurologic correlations}

Ryan A. Townley, MD, Bradley F. Boeve, MD, and Eduardo E. Benarroch, MD

Neurology ${ }^{\circledR}$ 2018;90:118-125. doi:10.1212/WNL.0000000000004840
Correspondence

Dr. Benarroch

benarroch.eduardo@

mayo.edu

Progranulin is a highly conserved secreted protein that is expressed in multiple cell types, both in the CNS and in peripheral tissues. Both directly and via its conversion to granulins, progranulin regulates cell growth, survival, repair, and inflammation. Progranulin has a major role in regulation of lysosomal function and microglial responses in the CNS. Autosomal dominant mutations of the progranulin (GRN) gene leading to protein haploinsufficiency are linked to familial frontotemporal dementia with neuropathologic frontotemporal lobar degeneration (FTLD) associated with accumulation of TAR-DNA binding protein of 43kDA (TDP-43) inclusions (FTLD-TDP). Homozygous GRN mutations are linked to neuronal ceroid lipofuscinosis (NCL). These findings have stimulated interest in elucidating the normal regulation and function of progranulin and granulins and the pathomechanisms by which progranulin deficiency leads to neurodegeneration. These topics have been recently reviewed ${ }^{1-5}$ and some salient aspects are discussed here.

\section{Representative case}

A 67-year-old right-handed man was evaluated for progressive forgetfulness, word-finding difficulties (particularly for nouns), and apathy over the previous 3 years. On mental status examination, he exhibited difficulties with attention, calculations, and delayed recall. There were no parkinsonian or motor neuron disease findings on examination. Difficulties with problem-solving manifested 5 years after symptom onset, forcing his retirement. Progression of fluent aphasia symptoms predominated over the next few years, with episodic memory less affected. At his final evaluation, 6 months prior to his death, he developed global aphasia, was dependent on activities of daily living, and had switched to his left hand for tasks due to severe apraxia of his dominant hand. He had preserved social graces, humor, and enjoyment for music. He died at age 76 years, 12 years after initial symptom onset. Corresponding MRI and FDGPET neuroimaging findings are shown in figure 1. Family history was relevant for dementia or parkinsonism in an autosomal dominant pattern affecting $>15$ relatives. Brain autopsy in several family members revealed TDP-43-positive neuronal intranuclear inclusions in the neocortex and striatum consistent with FTLD-TDP pathology type $1 .{ }^{6,7}$ Genetic sequencing in the progranulin (GRN) gene identified a heterozygous c.154delA. mutation in exon 3 of GRN in this patient and several affected members of this kindred.

\section{Comment}

This is a representative case of autosomal dominant FTLD (manifested in this patient primarily by progressive aphasia with subsequent mixed corticobasal syndrome features) due to heterozygous GRN mutations, as described in several series. ${ }^{8-16}$ The mechanism of neurodegeneration in this disorder is incompletely understood. The recognition that homozygous GRN mutations are associated with NCL, together with evidence from mouse models, suggest that neurodegeneration due to progranulin deficiency may reflect loss of its 


\section{Glossary}

FTLD = frontotemporal lobar degeneration; FTLD-TDP = frontotemporal lobar degeneration associated with accumulation of TAR-DNA binding protein of 43kDA; LAMP-1 = lysosome-associated membrane protein-1; NCL = neuronal ceroid lipofuscinosis; TDP-43 = TAR-DNA binding protein of 43kDA; TFEB = transcription factor EB.

effects as a neurotrophic factor, regulator of lysosomal function, inhibitor of excessive microglial activation, or combination thereof.

\section{Structure and regulation of progranulin}

\section{Structure}

Progranulin is a highly conserved secreted protein encoded by the GRN gene on human chromosome 17q21. Progranulin contains 7 and a half repeats of cysteine-rich granulin motifs separated by linker regions. ${ }^{4,17}$ A signal sequence allows progranulin to be secreted as a glycosylated protein that is proteolytically cleaved into $6 \mathrm{KDa}$ peptides (granulins A-G) (figure 2). Each granulin domain typically contains $12 \mathrm{cys}$ teine residues that form 6 disulfide bonds, which provide granulins with a compact $\beta$-sheet configuration. The cleavage of progranulin into granulins may be mediated by several proteases, including matrix metallopeptidases, disintegrin and matrix metalloproteases, neutrophil serine proteinase 3 , and elastases released from activated microglia. ${ }^{18}$ Progranulin may have effects on its own as a holoprotein or via its granulin products. Levels of progranulin and granulins may be regulated independently, either in the same or in opposite directions. Granulins oppose the effects of the holoprotein in inflammation ${ }^{4,19}$ and neuroprotection. ${ }^{20}$

\section{Expression and regulation}

Progranulin is expressed in many cell types throughout the body. ${ }^{21}$ In the CNS, it is produced by neurons, astrocytes, microglia, and endothelial cells. It is mainly expressed in neocortical neurons, granule and pyramidal cells of the hippocampus, Purkinje cells, ventromedial hypothalamus, and motor neurons ${ }^{21}$ and is upregulated in activated microglia. There are different GRN transcripts with either short or long $5^{\prime}$ untranslated regions; the presence of an upstream open reading frame in the longer $5^{\prime}$ untranslated region reduces mRNA stability and represses protein translation. ${ }^{22}$ MicroRNAs, such as miR-659, also regulate progranulin expression. ${ }^{23}$ Several stressors, such as hypoxia, glucose deprivation, acidosis, or oxidative stress, induce progranulin expression in vitro. The effects of hypoglycemia on GRN transcription involve p38 mitogen-activated protein kinase and downstream phosphorylation of transcription factors ${ }^{24}$; stimulation by hypoxia may be in part mediated by interactions of microRNA miR-659-3P with GRN transcripts. ${ }^{23}$ During synthesis, progranulin is translated directly into the endoplasmic reticulum and associates with disulfide isomerases yielding a mature protein with up to 44 disulfide bonds. ${ }^{25}$ Progranulin undergoes regulated glycosylation that protects the protein from lysosomal degradation.

\section{Trafficking and lysosomal degradation}

Progranulin is incorporated into vesicles and follows the secretory pathway, undergoing regulated exocytosis in an activity-dependent manner (figure 2). In neurons, progranulin is localized in dense-core vesicles and is cotransported with brain-derived neurotrophic factor by both anterograde and retrograde axonal transport. ${ }^{26}$ Neuronal activity increases the density of progranulin clusters along axons and increases progranulin recruitment at synapses. The basal secretion of progranulin in microglia is low; but upon activation, its secretion increases substantially. ${ }^{27}$ Once in the extracellular space, progranulin may undergo cleavage to granulins by action of extracellular protease ${ }^{28}$ or uptake into target cells via binding to sortilin- $1 .^{29}$ Sortilin-1 delivers progranulin via the endosome to the lysosome, where progranulin is cleaved and degraded. ${ }^{30}$ Progranulin has functional interactions with prosaposin, which is a secreted glycoprotein that has several similarities with progranulin. Like progranulin, prosaposin undergoes cleavage into cysteine-rich peptides called saposins and utilizes sortilin as a trafficking receptor to the lysosome, where saposins promote sphingolipid hydrolysis. ${ }^{31}$ Progranulin forms heterodimers with prosaposin; the interaction between these 2 proteins facilitates the lysosomal trafficking of each other. ${ }^{32}$ Prosaposin functions as a molecular bridge between progranulin and either the mannose-6 phosphate receptor or low-density lipoprotein receptor-related protein 1 in lysosomes, thereby providing a direct route for progranulin to reach this organelle. 33,34 Progranulin induces sortilin ubiquitination and internalization via clathrin-dependent endocytosis and sorting into early endosomes for lysosomal degradation. This constitutes a regulatory feedback mechanism whereby sortilin downregulation ensures sustained progranulin-mediated signaling and neuroprotection. ${ }^{35}$ Whereas glucose deprivation upregulates expression of progranulin expression, it reduces levels of sortilin. ${ }^{24}$

\section{Functions of progranulin}

Progranulin is a widely secreted growth factor regulating cell growth and survival, wound repair, and inflammation. ${ }^{16}$ Transcriptome analysis using RNA sequencing and bioinformatics in differentiated neuronal SH-SY5Y cells showed that both progranulin and the individual granulins elicit upregulation or downregulation of genes involved in 


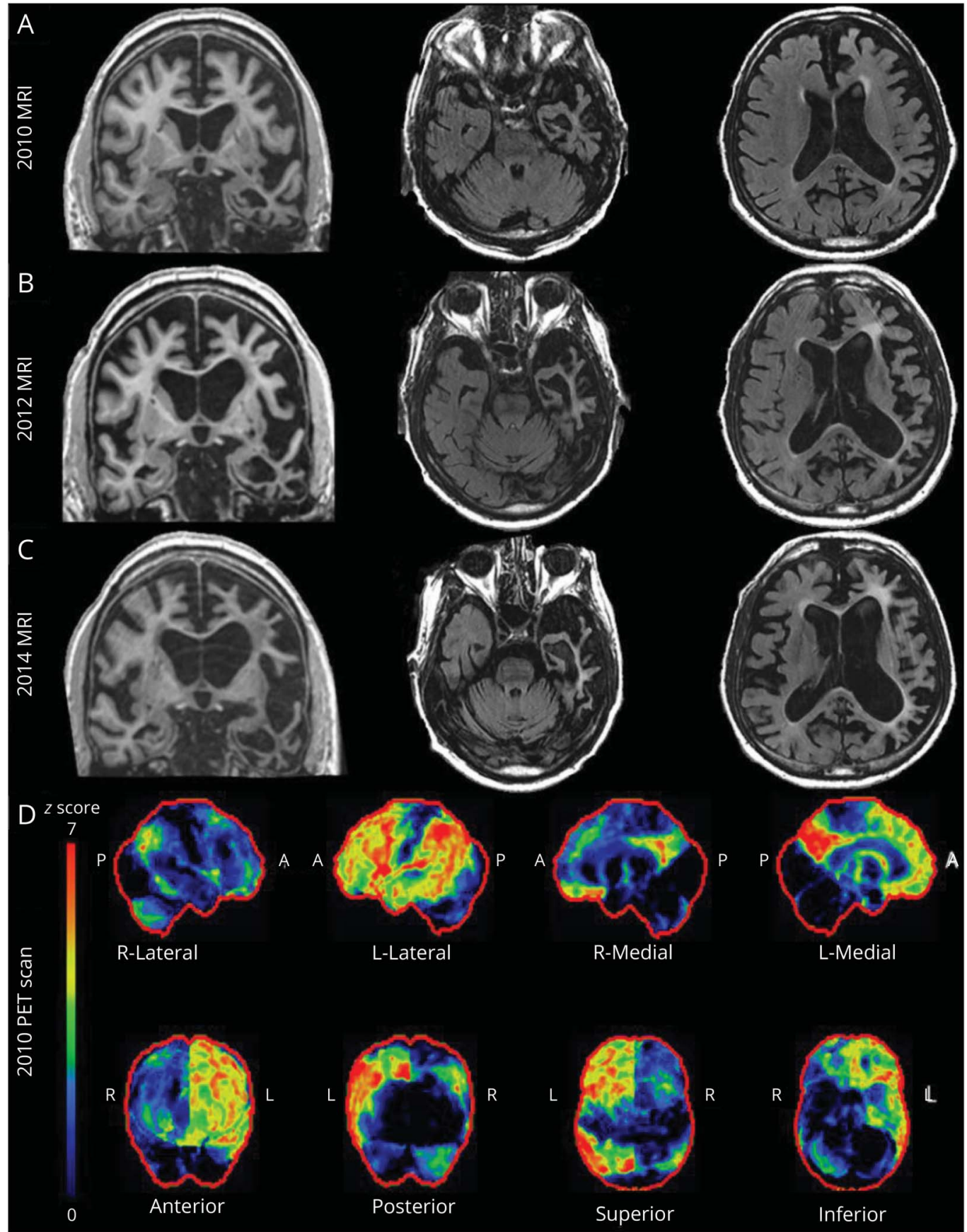

(A-C) MRI shows comparative coronal and axial T2-fluid-attenuated inversion recovery images 7, 9, and 11 years after symptom onset. (D) FDG-PET imaging 9 years after symptom onset shows dramatic asymmetric $L>R$ posterior cingulate cortex and precuneus hypometabolism along with asymmetric left frontal temporal, and parietal lobar hypometabolism.

transcriptional regulation, splicing, response to stress, endosomal sorting, cytoskeleton maintenance, and proteostasis. ${ }^{36}$

\section{Trophic and neuroprotective functions}

Progranulin and granulin E peptide promote survival and neurite outgrowth in neocortical, hippocampal, and motor neurons in vitro. ${ }^{37,38}$ Progranulin may act as an autocrine neurotrophic factor, as it is cotransported with brain-derived neurotrophic factor and secreted in an activity-dependent manner. Progranulin may exert neuroprotection by triggering survival signals involving phosphorylation cascades mediated by mitogen-activated kinases, extracellular signal related kinase, phosphatidylinositol 3'kinase, and glycogen synthase kinase- $3 \beta .^{37}$ The receptor mediating the neurotrophic effects 


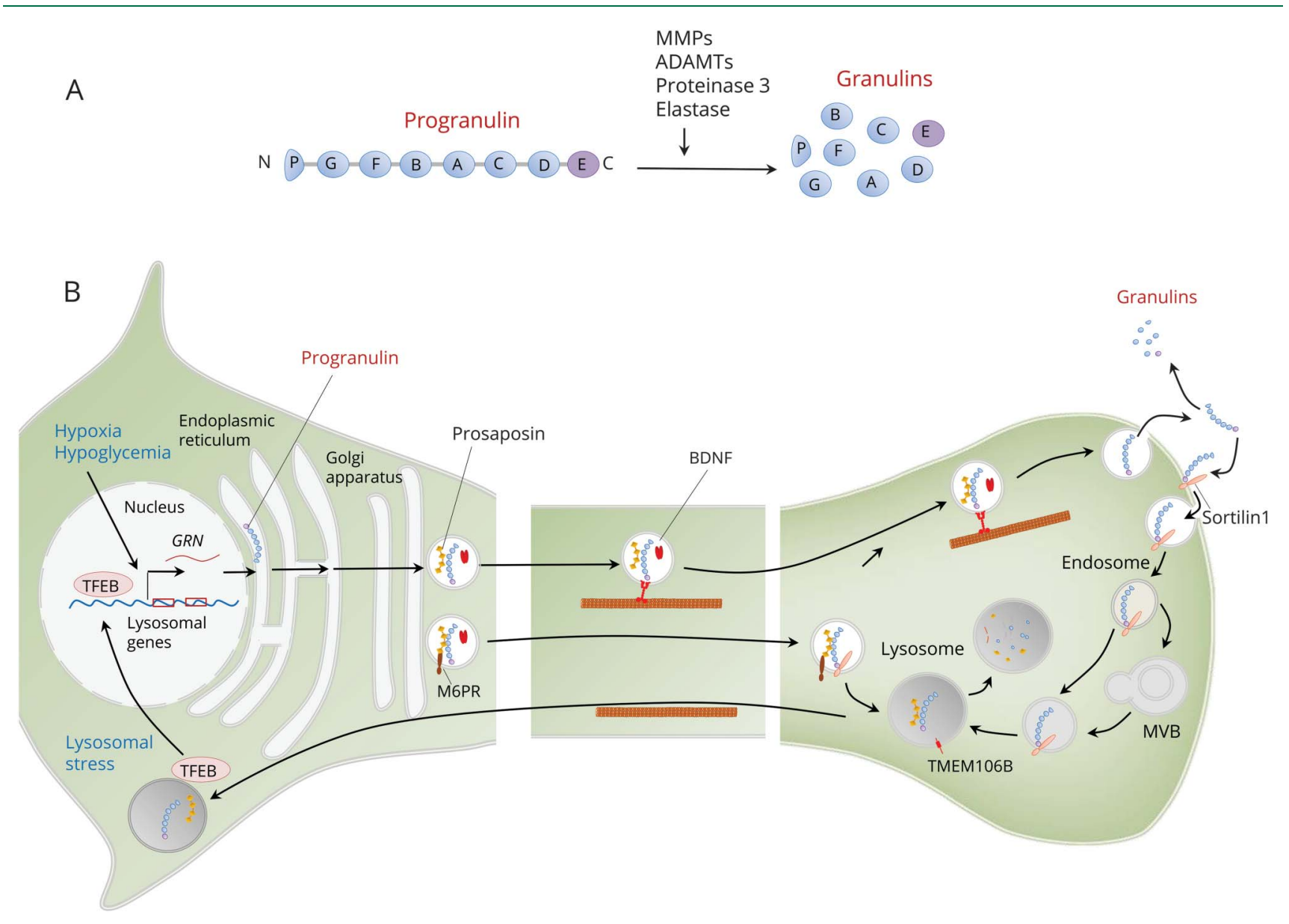

(A) Progranulin is encoded by the granulin (GRN) gene and contains 7.5 repeats of cysteine-rich granulin motifs separated by linker regions and is proteolytically cleaved into granulins A-G. The cleavage of progranulin into granulins may be mediated by several proteases, including matrix metallopeptidases (MMPs), a disintegrin and matrix metalloproteases (ADAMTs), neutrophil serine proteinase 3, and elastases released from activated microglia (B). Expression of the GRN gene increases in response to cellular stressors such a hypoxia and hypoglycemia. Once synthesized, progranulin follows the secretory pathway via dense-core vesicles where it is cotransported with brain-derived neurotrophic factor (BDNF) and prosaposin via axonal transport and then secreted by exocytosis. In the extracellular space, progranulin may either undergo cleavage to granulins or uptake into target cells via binding to sortilin-1. Sortilin-1 delivers progranulin via the endosome and multivesicular bodies (MVBs) to the lysosome, where progranulin is cleaved and degraded. Progranulin forms heterodimers with prosaposin, which promotes progranulin uptake in the lysosome via the mannose-6 phosphate receptor (M6PR). Progranulin regulates the formation and function of lysosomes, in part via the transcription factor EB (TFEB). In response to lysosomal stress, TFEB translocates to the nucleus and upregulates severe genes controlling lysosomal proteins, including progranulin. Transmembrane lysosomal protein 106B (TMEM106B) is located in the endosomes and lysosomes and opposes the effects of progranulin on lysosomal function.

of progranulin is yet to be identified. Progranulin binds to the ephrin-type A receptor 2 in the cell surface, ${ }^{39}$ leading to downstream activation of AKT (protein kinase B); this kinase is a common mediator of many effects of growth factor signals. ${ }^{40}$ Via this mechanism, progranulin promotes angiogenesis. Progranulin is also an endoplasmic reticulum stressresponsive factor. Endoplasmic reticulum stress increases progranulin expression, leading to activation of extracellular signal related kinase $1 / 2$ and AKT signaling through progranulin interaction with tumor necrosis factor receptor $2 .^{41}$ Activation of growth factor signal pathways by progranulin may rescue cortical neurons from cell death induced by glutamate or oxidative stress. Studies in experimental models of ischemia also show that progranulin regulates vascular permeability via vascular endothelial growth factor, suppresses neuroinflammation via interleukin 10 in microglia, and elicits neuroprotection in part by inhibition of cytoplasmic redistribution of TDP- $43 .^{42}$ In the periphery, progranulin binds perlecan, a major heparan sulfate proteoglycan of basement membranes and cell surfaces; this interaction could contribute to a fine regulation of tumor angiogenesis and affect cancer growth. ${ }^{43}$

\section{Lysosomal function}

There is evidence that progranulin regulates the formation and function of lysosomes. Progranulin colocalizes with the lysosome-associated membrane protein-1 (LAMP-1) and promotes lysosomal acidification by regulating levels of $\mathrm{V}$-type proton ATPase subunit d2; regulates levels of lysosomal proteins such as cathepsin $\mathrm{D}^{44}$; and binds to the transcription 
factor EB (TFEB), a master regulation of lysosomal biogenesis. ${ }^{45}$ Upon translocation from the lysosome to the nucleus, TFEB activates the coordinated lysosomal expression and regulation gene network as part of lysosomal stress response. ${ }^{45}$ This results in upregulation of genes encoding proteins involved in lysosomal biogenesis and function, including progranulin $^{46}$ and prosaposin. Transcriptome analysis shows that progranulin downregulates the charged multivesicular body proteins $1 \mathrm{~A}$ and 5 subunits of the endosomal sorting complex required for transport III. ${ }^{36}$ This complex is involved in internalization and transport of neuronal growth factors and signaling molecules and is one critical component of the endosomal-lysosomal pathway. ${ }^{47}$ Mutation of the charged multivesicular body protein $2 \mathrm{~B}$ subunit of this complex results in FTLD. ${ }^{48}$ Studies in Grn-/- knockout mice showed that progranulin deficiency is associated with increased immunoreactivity for LAMP-1 and increased expression of cathepsin-D, V-type ATPase subunit d2, and TFEB. ${ }^{49}$

\section{Microglial function}

Progranulin is a microglial chemoattractant and can increase endocytosis of extracellular peptides such as $\beta$-amyloid. ${ }^{50}$ In wild-type microglia, progranulin is localized to recycling endosomes, late endosomes, and early lysosomes, suggesting its role in regulating trafficking in these compartments. ${ }^{27}$ Progranulin may have anti-inflammatory properties via binding to tumor necrosis factor receptor 2, expressed on microglia. ${ }^{51}$ In contrast, granulins bind to and potentiate signaling from Toll-like receptor 9 in macrophages involved in innate immunity. ${ }^{52}$ Studies in Grn-/- knockout mice indicate that progranulin is required to suppress excessive microglial activation. Complete loss of progranulin causes aberrant increase in phagocytosis and secretion of proinflammatory cytokines by microglia. ${ }^{53-55}$ Transcriptome profiling in these mice shows that progranulin deficiency leads to an agedependent, progressive upregulation of lysosomal and innate immunity genes, including those encoding the complement components $\mathrm{C} 1 \mathrm{q}$ and $\mathrm{C} 3$; this is associated with enhanced synaptic pruning by microglia. ${ }^{27}$ With aging, Grn(-/-) mice developed marked microglia infiltration and preferential $\mathrm{Clq}$ promoted elimination of inhibitory synapses in the ventral thalamus, leading to thalamocortical excitability and obsessive-compulsive disorder-like grooming behavior. ${ }^{27}$

\section{Clinical correlations}

\section{Frontotemporal lobar degeneration}

The majority of patients with FTLD have accumulation of ubiquitinated proteins, primarily TDP-43 (FTLD-TDP), in the cytoplasm and nucleus. ${ }^{56}$ Mutations of the GRN gene on chromosome $17 \mathrm{q} 21$ are a common cause of FTLDTDP. ${ }^{6,8-11,16}$ These mutations include heterozygous frameshift, splice-site, and nonsense mutations and all result in GRN haploinsufficiency and loss of approximately $50 \%$ of functional progranulin. ${ }^{8-10}$ The clinical, neuroimaging, and neuropathologic features of FTLD-TDP due to GRN mutations have been the subject of various reviews ${ }^{12-15}$ and only few salient features are emphasized here. In general, this disorder manifests with behavioral or language difficulties, but the clinical manifestations vary widely among families and among individuals within individual families. Disease presentations may resemble primary progressive aphasia, ${ }^{10}$ corticobasal syndrome, ${ }^{57} \mathrm{Alz}$ heimer disease dementia (with prominent hippocampal sclerosis), ${ }^{58,59}$ or Lewy body disease. ${ }^{60}$ Mild parkinsonism is common but motor neuron disease is rare. ${ }^{12-14}$ The age at onset is also variable, and ranges from the 4th to the 9th decade. In contrast to mutations in the microtubule-associated protein tau (MAPT) and C9orf72 genes, there tends to be markedly asymmetric involvement of the frontal, temporal, and parietal lobes $^{61}$ (as shown in the representative case), and frontal white matter abnormalities can also be present. ${ }^{62}$ Neuropathologic features include frontal predominant atrophy with high density of TDP-43 inclusions in both the cytoplasm and nucleus. ${ }^{6}$ In the cortex, there is a predominant involvement of superficial cortical layers; the striatum may be prominently affected in some cases. $^{63}$

A genome-wide association study showed that single nucleotide polymorphisms (variants) of the TMEM106B gene on chromosome $7 \mathrm{p} 21$ encoding transmembrane lysosomal protein 106B may either increase ( $\mathrm{T}$ allele) or decrease ( $\mathrm{C}$ allele) the risk of developing FTLD-TDP. ${ }^{64}$ These polymorphisms also modify the risk of FTLD-TDP associated with chromosome 9 open reading frame 72 (C9orf72) gene expansion, as well as that of Alzheimer disease with hippocampal sclerosis. ${ }^{65}$ TMEM106B is a transmembrane glycoprotein predominantly located in the endosomes and lysosomes and its normal levels appear to be critical for lysosomal size, acidification, transport, and function. Increased TMEM106B expression promotes TFEB translocation to the nucleus (a marker of lysosomal stress) and reduces the number, maturation, and acidification of lysosomes causing a delay in endolysosome-dependent degradation. ${ }^{66}$ Studies in Grn-/- transgenic mice show that progranulin deficiency results in accumulation of TMEM106B as well as lysosomal abnormalities including lipofuscin accumulation during aging. ${ }^{67}$ This suggests that lysosomal dysfunction in progranulin deficiency may lead to abnormal processing of TDP-43 and other proteins. Cleaved granulins may also impair lysosomal degradation of proteins such as TDP- $43 .^{20}$

\section{Neuronal ceroid lipofuscinosis}

Homozygous GRN mutations cause NCL. ${ }^{3,11,68}$ NCL are primarily childhood neurodegenerative disorders associated with mutations in several genes (CLN1 to CLN14) encoding proteins with different cellular locations and functions. ${ }^{69}$ Many NCL proteins have been localized to lysosomes. The phenotype of CLN-11 linked to progranulin deficiency includes progressive visual loss, cerebellar ataxia, and myoclonic seizures developing between 20 and 30 years of age; skin biopsy shows the typical accumulation of vacuole-like organelles similar to other NCL forms. NCL-like features may also occur before the onset of dementia in patients with FTLD-TDP due to heterozygous GRN mutations. ${ }^{70}$ Noninvasive retinal imaging may reveal preclinical retinal lipofuscinosis, lymphoblasts accumulate 
prominent NCL material, and lysosomal inclusions may also be detected in cortical neurons at postmortem cortex. ${ }^{70}$ An NCL phenotype may also occur in siblings within families with neuropathologic confirmed FTLD. ${ }^{68}$

\section{Putative disease mechanisms}

As discussed above, models in knockout mice suggest that neurodegeneration associated with progranulin deficiency may reflect abnormal lysosomal function and exaggerated microglial inflammation. ${ }^{1}$ Mice lacking progranulin (Grn-/-) have impairment in social behaviors similar to those in patients with FTLD-TDP, lysosomal dysfunction, and neuroinflammation with microgliosis and astrocytosis in cortical and subcortical areas. ${ }^{71-73}$ These findings resemble those observed in NCL. Some evidence indicates that increased lipofuscinosis and gliosis may not be caused by intrinsic progranulin deficiency in neurons but rather involve microglia-derived progranulin. ${ }^{74}$ Heterozygous Grn+/- mice may represent a better model of GRN haploinsufficiency producing FTLD-TDP. However, unlike homozygous knockouts, these animals have age-dependent behavioral deficits without evidence of neuroinflammation or lipofuscinoses. ${ }^{75}$ One limitation of the mouse models developed so far is that they do not fully recapitulate the spectrum of TDP-43 neuropathology seen in FTLD-TDP, although some models show TDP-43 hyperphosphorylation. ${ }^{53,73,76}$ Consistent with an opposing interaction between progranulin and TMEM106 B in lysosomes, a recent study showed that loss of TMEM106 B due to Tmem16 $b$ deletion ameliorates lysosomal and frontotemporal dementia-related phenotypes in progranulin deficient mice. $^{77}$

Progranulin deficiency may result in accumulation of other proteins associated with neurodegeneration, besides TDP43. There are cases of FTLD-TDP due to GRN haploinsufficiency with accumulation of not only TDP-43 but also tau and synuclein inclusions in neurons and glial cells. $^{78,79}$ Progranulin protects against $\beta$-amyloid deposition and toxicity in experimental mouse models of Alzheimer disease. $^{80}$ Conceivably, progranulin deficiency, either through lysosomal autophagy dysfunction or other defects of proteostasis, may have a widespread effect on processing of abnormal proteins. The more widespread effects, or other factors, are likely at play since one would anticipate that a 50\% reduction in progranulin would affect the whole brain, or at least both hemispheres relatively equally, whereas a substantial proportion of heterozygous GRN mutation carriers have markedly focal/asymmetric imaging findings and associated clinical presentations.

\section{Therapeutic implications}

Given the potential neuroprotective role of progranulin and the consequences of progranulin deficiency, there have been several approaches aimed to increase cellular progranulin levels based on preclinical studies. These include enhancers of GRN transcription such as suberoylanilide hydroxamic acid (SAHA, vorinostat $)^{81}$; blockers of extracellular receptor kinase pathways such as selumetinib (AZD6244) ${ }^{82}$; alkalinizing inhibitors of progranulin lysosomal degradation that target vacuolar $\mathrm{H}^{+}$ ATPase, such as bafilomycin $\mathrm{Al}^{30}$ or amiodarone ${ }^{83}$; and sotrtilin-1 antagonists and small-molecule progranulin binders to inhibit progranulin endocytosis. ${ }^{84}$ These studies so far have provided negative results. A clinical trial (clinicaltrials.gov/ show/NCT02149160) involving a histone deacetylase inhibitor in GRN mutation carriers was terminated prematurely due to financial limitations; the safety and efficacy data have not yet been published. Plasma progranulin levels are lower in GRN mutation carriers than in controls, suggesting that they could be used for monitoring therapeutic response to progranulinenhancing agents. ${ }^{85}$ However, the variable source and dynamic regulation of progranulin may explain the poor correlation between its concentrations in plasma and CSF. ${ }^{86}$

\section{Perspective}

Progranulin and its degradation products, granulins, have widespread and fundamental effects in several aspects of cell biology. In addition to its effects in the CNS, progranulin promotes growth and chemotherapy resistance of neoplasms, and functions as an adipokine mediating obesity and insulin resistance. ${ }^{87}$ Where loss of progranulin function in some forms of FTLD-TDP and NCL provide a rationale for attempts to increase progranulin levels as a neuroprotective approach to neurodegenerative disorders, such attempts may be hindered by the peripheral effects of progranulin in promoting carcinogenesis and obesity. Target selective approaches may be necessary to fully determine the potential benefit of upregulating progranulin signaling in the CNS.

\section{Author contributions}

$\mathrm{R}$. Townley: drafting and revising the manuscript, analysis of data. B. Boeve: design and conceptualization, analysis of data, revising the manuscript. E. Benarroch: design and conceptualization, analysis of data, drafting the manuscript, and revising the manuscript for intellectual content.

\section{Study funding}

Supported in part by U01 AG045390, U54 NS092089, and P50 AG016574.

\section{Disclosure}

R. Townley reports no disclosures. B. Boeve has served as an investigator for clinical trials sponsored by GE Healthcare and Axovant; receives royalties from the publication of a book titled Behavioral Neurology of Dementia (Cambridge Medicine, 2009, 2016); serves on the Scientific Advisory Board of the Tau Consortium; and receives research support from the NIH (U01 AG045390, U54 NS092089, P50 AG016574, UO1 AG006786, RO1 AG041797), the Mayo Clinic Dorothy and Harry T. Mangurian Jr. Lewy Body Dementia Program, and the Little Family Foundation. E. Benarroch receives a stipend 
in his capacity as section editor of Clinical Implications of Neuroscience Research for Neurology ${ }^{\circledR}$. Go to Neurology.org/N for full disclosures.

\section{References}

1. Kao AW, McKay A, Singh PP, Brunet A, Huang EJ. Progranulin, lysosomal regulation and neurodegenerative disease. Nat Rev Neurosci 2017;18:325-333.

2. Cenik B, Sephton CF, Kutluk Cenik B, Herz J, Yu G. Progranulin: a proteolytically processed protein at the crossroads of inflammation and neurodegeneration. J Biol Chem 2012;287:32298-32306.

3. Smith KR, Damiano J, Franceschetti S, et al. Strikingly different clinicopathological phenotypes determined by progranulin-mutation dosage. Am J Hum Genet 2012;90: 1102-1107.

4. De Muynck L, Van Damme P. Cellular effects of progranulin in health and disease. J Mol Neurosci 2011;45:549-560.

5. Eriksen JL. The enigmatic roles of microglial versus neuronal progranulin in neurological disease. Acta Neuropathol 2010;119:107-109.

6. Mackenzie IR. The neuropathology and clinical phenotype of FTD with progranulin mutations. Acta Neuropathol 2007;114:49-54.

7. Josephs KA, Ahmed Z, Katsuse O, et al. Neuropathologic features of frontotemporal lobar degeneration with ubiquitin-positive inclusions with progranulin gene (PGRN) mutations. J Neuropathol Exp Neurol 2007;66:142-151.

8. Cruts M, Gijselinck I, van der Zee J, et al. Null mutations in progranulin cause ubiquitin-positive frontotemporal dementia linked to chromosome 17q21. Nature 2006;442:920-924

9. Gass J, Cannon A, Mackenzie IR, et al. Mutations in progranulin are a major cause of ubiquitin-positive frontotemporal lobar degeneration. Hum Mol Genet 2006;15: 2988-3001.

10. Snowden JS, Pickering-Brown SM, Mackenzie IR, et al. Progranulin gene mutations associated with frontotemporal dementia and progressive non-fluent aphasia. Brain 2006;129:3091-3102.

11. Baker M, Mackenzie IR, Pickering-Brown SM, et al. Mutations in progranulin cause tau-negative frontotemporal dementia linked to chromosome 17. Nature 2006;442: 916-919.

12. Boeve BF, Baker M, Dickson DW, et al. Frontotemporal dementia and parkinsonism associated with the IVS1+1G->A mutation in progranulin: a clinicopathologic study. Brain 2006;129:3103-3114.

13. van Swieten JC, Heutink P. Mutations in progranulin (GRN) within the spectrum of clinical and pathological phenotypes of frontotemporal dementia. Lancet Neurol 2008;7:965-974.

14. Kelley BJ, Haidar W, Boeve BF, et al. Prominent phenotypic variability associated with mutations in progranulin. Neurobiol Aging 2009;30:739-751.

15. Cerami C, Scarpini E, Cappa SF, Galimberti D. Frontotemporal lobar degeneration: current knowledge and future challenges. J Neurol 2012;259:2278-2286.

16. Nicholson AM, Gass J, Petrucelli L, Rademakers R. Progranulin axis and recent developments in frontotemporal lobar degeneration. Alzheimers Res Ther 2012; $4: 4$.

17. Bhandari V, Palfree RG, Bateman A. Isolation and sequence of the granulin precursor cDNA from human bone marrow reveals tandem cysteine-rich granulin domains. Proc Natl Acad Sci USA 1992;89:1715-1719.

18. Bateman A, Bennett HP. The granulin gene family: from cancer to dementia. Bioessays 2009;31:1245-1254.

19. Kessenbrock K, Frohlich L, Sixt M, et al. Proteinase 3 and neutrophil elastase enhance inflammation in mice by inactivating antiinflammatory progranulin. J Clin Invest 2008;118:2438-2447.

20. Salazar DA, Butler VJ, Argouarch AR, et al. The progranulin cleavage products, granulins, exacerbate TDP-43 toxicity and increase TDP-43 levels. J Neurosci 2015; 35:9315-9328.

21. Daniel R, He Z, Carmichael KP, Halper J, Bateman A. Cellular localization of gene expression for progranulin. J Histochem Cytochem 2000;48:999-1009.

22. Capell A, Fellerer K, Haass C. Progranulin transcripts with short and long 5' untranslated regions (UTRs) are differentially expressed via posttranscriptional and translational repression. J Biol Chem 2014;289:25879-25889.

23. Piscopo P, Grasso M, Fontana F, et al. Reduced miR-659-3p levels correlate with progranulin increase in hypoxic conditions: implications for frontotemporal dementia. Front Mol Neurosci 2016;9:31.

24. Kawashima KI, Ishiuchi Y, Konnai M, et al. Glucose deprivation regulates the progranulin-sortilin axis in PC12 cells. FEBS Open Bio 2017;7:149-159.

25. Almeida S, Zhou L, Gao FB. Progranulin, a glycoprotein deficient in frontotemporal dementia, is a novel substrate of several protein disulfide isomerase family proteins. PLoS One 2011;6:e26454.

26. Petoukhov E, Fernando S, Mills F, et al. Activity-dependent secretion of progranulin from synapses. J Cell Sci 2013;126:5412-5421.

27. Lui H, Zhang J, Makinson SR, et al. Progranulin deficiency promotes circuit-specific synaptic pruning by microglia via complement activation. Cell 2016;165:921-935.

28. Suh HS, Choi N, Tarassishin L, Lee SC. Regulation of progranulin expression in human microglia and proteolysis of progranulin by matrix metalloproteinase-12 (MMP-12). PLoS One 2012;7:e35115.

29. Hu F, Padukkavidana T, Vaegter CB, et al. Sortilin-mediated endocytosis determines levels of the frontotemporal dementia protein, progranulin. Neuron 2010;68: 654-667.
30. Capell A, Liebscher S, Fellerer K, et al. Rescue of progranulin deficiency associated with frontotemporal lobar degeneration by alkalizing reagents and inhibition of vacuolar ATPase. J Neurosci 2011;31:1885-1894.

31. Meyer RC, Giddens MM, Coleman BM, Hall RA. The protective role of prosaposin and its receptors in the nervous system. Brain Res 2014;1585:1-12.

32. Zhou X, Sullivan PM, Sun L, Hu F. The interaction between progranulin and prosaposin is mediated by granulins and the linker region between saposin B and C. J Neurochem 2017;143:236-243.

33. Zhou X, Sun L, Bastos de Oliveira F, et al. Prosaposin facilitates sortilin-independent lysosomal trafficking of progranulin. J Cell Biol 2015;210:991-1002.

34. Nicholson AM, Finch NA, Almeida M, et al. Prosaposin is a regulator of progranulin levels and oligomerization. Nat Commun 2016;7:11992.

35. Tanimoto R, Palladino C, Xu SQ, et al. The perlecan-interacting growth factor progranulin regulates ubiquitination, sorting, and lysosomal degradation of sortilin. Matrix Biol 2017;S0945-053X:30108-30117.

36. Rollinson S, Young K, Bennion-Callister J, Pickering-Brown SM. Identification of biological pathways regulated by PGRN and GRN peptide treatments using transcriptome analysis. Eur J Neurosci 2016;44:2214-2225.

37. Gao X, Joselin AP, Wang L, et al. Progranulin promotes neurite outgrowth and neuronal differentiation by regulating GSK-3beta. Protein Cell 2010;1:552-562.

38. Gass J, Lee WC, Cook C, et al. Progranulin regulates neuronal outgrowth independent of sortilin. Mol Neurodegener 2012;7:33.

39. Chitramuthu B, Bateman A. Progranulin and the receptor tyrosine kinase EphA2, partners in crime? J Cell Biol 2016;215:603-605

40. Neill T, Buraschi S, Goyal A, et al. EphA2 is a functional receptor for the growth factor progranulin. J Cell Biol 2016;215:687-703.

41. Li M, Liu Y, Xia F, et al. Progranulin is required for proper ER stress response and inhibits ER stress-mediated apoptosis through TNFR2. Cell Signal 2014;26: $1539-1548$

42. Kanazawa M, Kawamura K, Takahashi T, et al. Multiple therapeutic effects of progranulin on experimental acute ischaemic stroke. Brain 2015;138:1932-1948.

43. Gonzalez EM, Mongiat M, Slater SJ, Baffa R, Iozzo RV. A novel interaction between perlecan protein core and progranulin: potential effects on tumor growth. J Biol Chem 2003;278:38113-38116.

44. Tanaka Y, Suzuki G, Matsuwaki T, et al. Progranulin regulates lysosomal function and biogenesis through acidification of lysosomes. Hum Mol Genet 2017;26:969-988.

45. Sardiello M, Palmieri M, di Ronza A, et al. A gene network regulating lysosomal biogenesis and function. Science 2009;325:473-477.

46. Belcastro V, Siciliano V, Gregoretti F, et al. Transcriptional gene network inference from a massive dataset elucidates transcriptome organization and gene function. Nucleic Acids Res 2011;39:8677-8688.

47. Bronfman FC, Escudero CA, Weis J, Kruttgen A. Endosomal transport of neurotrophins: roles in signaling and neurodegenerative diseases. Dev Neurobiol 2007;67: 1183-1203.

48. Skibinski G, Parkinson NJ, Brown JM, et al. Mutations in the endosomal ESCRTIIIcomplex subunit CHMP2B in frontotemporal dementia. Nat Genet 2005;37: 806-808.

49. Tanaka Y, Chambers JK, Matsuwaki T, Yamanouchi K, Nishihara M. Possible in volvement of lysosomal dysfunction in pathological changes of the brain in aged progranulin-deficient mice. Acta Neuropathol Commun 2014;2:78.

50. Pickford F, Marcus J, Camargo LM, et al. Progranulin is a chemoattractant for microglia and stimulates their endocytic activity. Am J Pathol 2011;178. 284-295

51. Galimberti D, Bonsi R, Fenoglio C, et al. Inflammatory molecules in Frontotemporal Dementia: cerebrospinal fluid signature of progranulin mutation carriers. Brain Behav Immun 2015;49:182-187.

52. Park B, Buti L, Lee S, et al. Granulin is a soluble cofactor for toll-like receptor signaling. Immunity 2011;34:505-513.

53. Yin F, Banerjee R, Thomas B, et al. Exaggerated inflammation, impaired host defense, and neuropathology in progranulin-deficient mice. J Exp Med 2010;207:117-128.

54. Kao AW, Eisenhut RJ, Martens LH, et al. A neurodegenerative disease mutation that accelerates the clearance of apoptotic cells. Proc Natl Acad Sci USA 2011;108: 4441-4446.

55. Martens LH, Zhang J, Barmada SJ, et al. Progranulin deficiency promotes neuroinflammation and neuron loss following toxin-induced injury. J Clin Invest 2012;122: 3955-3959.

56. Arai T, Hasegawa M, Akiyama H, et al. TDP-43 is a component of ubiquitin-positive tau-negative inclusions in frontotemporal lobar degeneration and amyotrophic latera sclerosis. Biochem Biophys Res Commun 2006;351:602-611.

57. Spina S, Murrell JR, Huey ED, et al. Corticobasal syndrome associated with the A9D progranulin mutation. J Neuropathol Exp Neurol 2007;66:892-900.

58. Perry DC, Lehmann M, Yokoyama JS, et al. Progranulin mutations as risk factors for Alzheimer disease. JAMA Neurol 2013;70:774-778.

59. Kelley BJ, Haidar W, Boeve BF, et al. Alzheimer disease-like phenotype associated with the c.154delA mutation in progranulin. Arch Neurol 2010;67:171-177.

60. Arosio B, Abbate C, Galimberti D, et al. GRN Thr272fs clinical heterogeneity: a case with atypical late onset presenting with a dementia with Lewy bodies phenotype. J Alzheimers Dis 2013;35:669-674.

61. Whitwell JL, Weigand SD, Boeve BF, et al. Neuroimaging signatures of frontotemporal dementia genetics: C9ORF72, tau, progranulin and sporadics. Brain 2012 135:794-806.

62. Ameur F, Colliot O, Caroppo P, et al. White matter lesions in FTLD: distinct phenotypes characterize GRN and C9ORF72 mutations. Neurol Genet 2016;2: e47. 
63. Davion S, Johnson N, Weintraub S, et al. Clinicopathologic correlation in PGRN mutations. Neurology 2007;69:1113-1121.

64. Van Deerlin VM, Sleiman PM, Martinez-Lage M, et al. Common variants at $7 \mathrm{p} 21$ are associated with frontotemporal lobar degeneration with TDP-43 inclusions. Nat Genet 2010;42:234-239.

65. Nicholson AM, Rademakers R. What we know about TMEM106B in neurodegeneration. Acta Neuropathol 2016;132:639-651.

66. Brady OA, Zheng Y, Murphy K, Huang M, Hu F. The frontotemporal lobar degeneration risk factor, TMEM106B, regulates lysosomal morphology and function. Hum Mol Genet 2013;22:685-695.

67. Zhou X, Sun L, Brady OA, Murphy KA, Hu F. Elevated TMEM106B levels exaggerate lipofuscin accumulation and lysosomal dysfunction in aged mice with progranulin deficiency. Acta Neuropathol Commun 2017;5:9.

68. Almeida MR, Macario MC, Ramos L, Baldeiras I, Ribeiro MH, Santana I. Portuguese family with the co-occurrence of frontotemporal lobar degeneration and neuronal ceroid lipofuscinosis phenotypes due to progranulin gene mutation. Neurobiol Aging 2016;41:e201-e205.

69. Carcel-Trullols J, Kovacs AD, Pearce DA. Cell biology of the NCL proteins: what they do and don't do. Biochim Biophys Acta 2015;1852:2242-2255.

70. Ward ME, Chen R, Huang HY, et al. Individuals with progranulin haploinsufficiency exhibit features of neuronal ceroid lipofuscinosis. Sci Transl Med 2017;9:eaah5642.

71. Kayasuga Y, Chiba S, Suzuki M, et al. Alteration of behavioural phenotype in mice by targeted disruption of the progranulin gene. Behav Brain Res 2007;185:110-118.

72. Kleinberger G, Capell A, Haass C, Van Broeckhoven C. Mechanisms of granulin deficiency: lessons from cellular and animal models. Mol Neurobiol 2013;47:337-360.

73. Gotzl JK, Mori K, Damme M, et al. Common pathobiochemical hallmarks of progranulin-associated frontotemporal lobar degeneration and neuronal ceroid lipofuscinosis. Acta Neuropathol 2014;127:845-860.

74. Petkau TL, Blanco J, Leavitt BR. Conditional loss of progranulin in neurons is not sufficient to cause neuronal ceroid lipofuscinosis-like neuropathology in mice. Neurobiol Dis 2017;106:14-22.

75. Filiano AJ, Martens LH, Young AH, et al. Dissociation of frontotemporal dementiarelated deficits and neuroinflammation in progranulin haploinsufficient mice. J Neurosci 2013;33:5352-5361.
76. Wils H, Kleinberger G, Pereson S, et al. Cellular ageing, increased mortality and FTLD-TDP-associated neuropathology in progranulin knockout mice. J Pathol 2012; 228:67-76.

77. Klein ZA, Takahashi H, Ma M, et al. Loss of TMEM106B ameliorates lysosomal and frontotemporal dementia-related phenotypes in progranulin-deficient mice. Neuron 2017;95:281-296 e286.

78. Leverenz JB, Yu CE, Montine TJ, et al. A novel progranulin mutation associated with variable clinical presentation and tau, TDP43 and alpha-synuclein pathology. Brain 2007;130:1360-1374.

79. Hosokawa M, Kondo H, Serrano GE, et al. Accumulation of multiple neurodegenerative disease-related proteins in familial frontotemporal lobar degeneration associated with granulin mutation. Sci Rep 2017;7:1513

80. Minami SS, Min SW, Krabbe G, et al. Progranulin protects against amyloid beta deposition and toxicity in Alzheimer's disease mouse models. Nat Med 2014;20: $1157-1164$.

81. Cenik B, Sephton CF, Dewey CM, et al. Suberoylanilide hydroxamic acid (vorinostat) up-regulates progranulin transcription: rational therapeutic approach to frontotemporal dementia. J Biol Chem 2011;286:16101-16108.

82. Alquezar C, Esteras N, de la Encarnacion A, Moreno F, Lopez de Munain A, MartinRequero A. Increasing progranulin levels and blockade of the ERK1/2 pathway: upstream and downstream strategies for the treatment of progranulin deficient frontotemporal dementia. Eur Neuropsychopharmacol 2015;25:386-403.

83. Alberici A, Archetti S, Pilotto A, et al. Results from a pilot study on amiodarone administration in monogenic frontotemporal dementia with granulin mutation. Neurol Sci 2014;35:1215-1219.

84. Lee WC, Almeida S, Prudencio M, et al. Targeted manipulation of the sortilinprogranulin axis rescues progranulin haploinsufficiency. Hum Mol Genet 2014;23: $1467-1478$

85. Meeter LH, Patzke H, Loewen G, et al. Progranulin levels in plasma and cerebrospinal fluid in granulin mutation carriers. Dement Geriatr Cogn Dis Extra 2016;6:330-340.

86. Nicholson AM, Finch NA, Thomas CS, et al. Progranulin protein levels are differently regulated in plasma and CSF. Neurology 2014;82:1871-1878.

87. Abella V, Pino J, Scotece M, et al. Progranulin as a biomarker and potential therapeutic agent. Drug Discov Today 2017;22:1557-1564.

\section{Neurology $y^{\circledR}$ Online CME Program}

Earn CME while reading Neurology. This program is available only to online Neurology subscribers. Read the articles marked CME, go to Neurology.org, and click on CME. This will provide all of the information necessary to get started. The American Academy of Neurology (AAN) is accredited by the Accreditation Council for Continuing Medical Education (ACCME) to sponsor continuing medical education for physicians. Neurology is planned and produced in accordance with the ACCME Essentials. For more information, contact AAN Member Services at 800-879-1960.

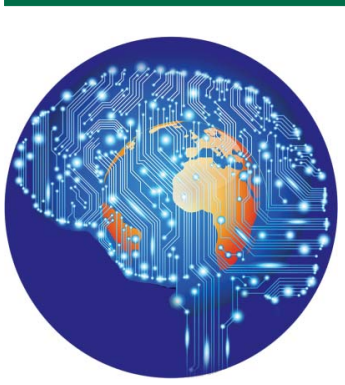

\section{Practice Current: An interactive exchange on controversial topics}

Share your own best practices.

Read commentary with expert opinion.

Explore results on an interactive world map.

NPub.org/NCP/practicecurrent

Neurology ${ }^{\circledR}$ Clinical Practice 